\title{
Cirugía en pacientes con pancreatitis aguda: serie de casos en un hospital de tercer nivel
}

\section{Surgery in patients with acute pancreatitis: third level hospital case series}

\author{
Carlos A. Santana-Pérez*, Yisvanth Pérez-Ponce y Luis Á. Muciño-Pérez \\ Departamento de Cirugía General, Cirugía del aparato digestivo, Centro Médico ISSEMyM Lic. Arturo Montiel Rojas, Toluca, Méx., México
}

\begin{abstract}
Resumen
La incidencia de pancreatitis aguda alcanza hasta 80 por cada 100,000 habitantes. La litiasis biliar es el origen más frecuente de la pancreatitis, sus complicaciones inmediatas y mediatas: necrosis infectada, colecciones o absceso, pueden ocasionar incluso la muerte. Objetivo: Descripción de serie de casos. Material y métodos: Revisión de los archivos clínicos de pacientes con diagnóstico de pancreatitis aguda grave en el periodo 2013 al 2019 en el Centro Médico ISSEMyM Toluca. Se analizaron: edad, comorbilidades, etiología, diagnóstico tomográfico, abordaje quirúrgico, frecuencia de complicaciones y tasa de mortalidad. Resultados: Cincuenta y nueve pacientes con diagnóstico de pancreatitis aguda severa de los cuales 30 se excluyeron. Se incluyeron 29 pacientes, 16 hombres (56\%) y 13 mujeres (44\%). Veintisiete pacientes contaban con reporte de litiasis vesicular (92.5\%) y 2 de hipertrigliceridemia (7.4\%). A 29 (100\%) se les realizó tomografía axial computarizada. De 29 pacientes, 18 contaron con diagnóstico de necrosis pancreática y colección o absceso aunado a respuesta inflamatoria sistémica. A 14 (55\%) se les realizó laparotomía exploradora, 12 (44.3\%) laparoscopia exploradora y $2(0.7 \%)$ drenaje percutáneo. Se reportaron dos defunciones. Conclusiones: El manejo de las complicaciones debe ser llevado de forma oportuna con recursos adecuados, a fin de evitar un desenlace fatal en los pacientes con pancreatitis.
\end{abstract}

Palabras clave: Pancreatitis. Pseudoquiste. Necrosis infectada. Laparotomía. Laparoscopia. Cistoyeyuno anastomosis.

\section{Abstract}

The incidence of acute pancreatitis reaches up to 80 per 100,000 inhabitants. Biliary lithiasis most frequent origin of pancreatitis. Its immediate and mediate complications; infected necrosis, collections or abscess, can even cause death. Objective: Description of case series. Material and methods: Review of the clinical files of patients diagnosed with severe acute pancreatitis in the period 2013 to 2019 at the ISSEMyM Toluca Medical Center. Analyzing; age, comorbidities, etiology, tomographic diagnosis, surgical approach, frequency of complications and mortality rate. Results: 59 patients diagnosed with severe acute pancreatitis of which 30 were excluded. 29 patients, 16 men (56\%) and 13 women (44\%) were included. 27 patients had a report of gallbladder lithiasis (92.5\%) and 2 of hypertriglyceridemia (7.4\%). 29 (100\%) underwent computed axial tomography. Of the total (29 patients), 18 had a diagnosis of pancreatic necrosis and a collection or abscess combined with a systemic inflammatory response. 14 (55\%) underwent exploratory laparotomy, 12 (44.3\%) exploratory laparoscopy, and $2(0.7 \%)$ percutaneous drainage. 2 deaths were reported. Conclusions: The management of complications must be carried out in a timely manner with adequate resources, in order to avoid a fatal outcome in patients with pancreatitis.

Key words: Acute pancreatitis. Pseudocyst. Infected necrosis. Laparotomy. Laparoscopy. Cystojejunoanastomosis. Cystogastro anastomosis. 


\section{Introducción}

La importancia de la pancreatitis aguda (PA) no es poco considerable y los estudios epidemiológicos observaron que su incidencia es de alrededor de 5 a 80 por 100,000 en una población. La prevalencia de PA es muy variable en todo el mundo e incluso se pueden ver grandes diferencias en diferentes áreas de un país determinado.

La PA es un trastorno inflamatorio agudo del páncreas que afecta al páncreas y los tejidos peripancreáticos, pero a veces también puede afectar a otros sistemas orgánicos. La tasa de complicaciones entre estos pacientes varía entre el 10 y el $20 \%$ y si no se trata de inmediato, la mortalidad puede aumentar hasta el $25 \%$. Aunque la mayoría de los pacientes resuelve de forma espontánea.

Lascausasmás comunes de PA son los cálculos biliaresy el abuso del alcohol, que representan el $70-80 \%$ de los casos. Otros factores de riesgo incluyen: hipertrigliceridemia, hipercalcemia, medicamentos, infecciones, traumas, colangiopancreatografía retrógrada endoscópica posterior, tumores y divisismo pancreático, y algunos casos también son idiopáticos ${ }^{1,2}$.

Losfactoresasociadosa la gravedad son necrosis, infeccióny falla orgánica múltiple. Latendencia de la mortalidad muestra una curva bifásica. En la primera semana se relaciona directamente con el desarrollo temprano de falla multiorgánica. El segundo pico de mortalidad se asocia con sepsis secundaria a necrosis infectada ${ }^{3,4}$.

Aunque la tecnología de diagnóstico y tratamiento para la PA se ha desarrollado rápidamente, el pronóstico de la PA grave todavía es malo. Una cirugía de desbridamiento realizada demasiado temprano para la pancreatitis necrotizante puede agravar los síntomas. El trauma de la laparotomía es mayor, la incidencia de complicaciones postoperatorias es aproximadamente del 34 al 95\% y la mortalidad es del 11 al $39 \%$.

La necrosis pancreática infectada (NPI) representa el $33 \%$ de la pancreatitis necrotizante. La mortalidad en pacientes con NPI es de alrededor del $32 \%$. En los últimos años se ha alcanzado un consenso básicamente en cuanto al momento en que se realizará el tratamiento de la NPI. En general, se cree que la intervención quirúrgica debe realizarse aproximadamente cuatro semanas después del inicio de los síntomas ${ }^{6}$.

A pesar de conocer las causas etiológicas de la PA que podrían tratarse con cirugía (litiasis vesicular, hiperparatiroidismo y traumatismo), las indicaciones más comunes para el tratamiento quirúrgico en la PA son necrosis pancreática y peripancreática infectadas
Tabla 1. Criterios de inclusión y exclusión

\begin{tabular}{|c|c|}
\hline Criterios de inclusión & Criterios de exclusión \\
\hline $\begin{array}{l}\text { 1. Paciente con diagnóstico de } \\
\text { pancreatitis aguda (CIE-10 K 85) } \\
\text { 2. Pacientes con diagnóstico de } \\
\text { severidad Ranson mayor a } 3 \text { al } \\
\text { ingreso y APACHE II mayor de } 8 \\
\text { puntos } \\
\text { 3. Paciente con diagnóstico } \\
\text { tomográfico Balthazar E y D con } \\
\text { necrosis, colección y abscesos } \\
\text { peripancreáticos infectados } \\
\text { sugerido por presentar } \\
\text { procalcitonina } 2 \text { a } 10 \text { ng/dl, signos } \\
\text { y síntomas de respuesta } \\
\text { inflamatoria } \\
\text { 4. Pacientes con diagnóstico inicial } \\
\text { de pancreatitis aguda severa que } \\
\text { desarrollaron pseudoquiste } \\
\text { pancreático a las } 4 \text { semanas } \\
\text { 5. Pacientes intervenidos } \\
\text { quirúrgicamente en el Centro } \\
\text { Médico ISSEMYM Toluca en el } \\
\text { periodo del } 2013 \text { al } 2019\end{array}$ & $\begin{array}{l}\text { 6. Pacientes cuyos } \\
\text { expedientes no } \\
\text { contaran con lo } \\
\text { establecido en la } \\
\text { NOM-004-SSA3-2012 } \\
\text { 7. Pacientes } \\
\text { postoperados en } \\
\text { unidad externa a } \\
\text { esta institución } \\
\text { 8. Pacientes con } \\
\text { diagnóstico diferente } \\
\text { a pancreatitis aguda } \\
\text { (CIE-10 K 85) } \\
\text { 9. Pacientes } \\
\text { postoperados que no } \\
\text { tuvieran escala de } \\
\text { severidad Ranson } \\
\text { mayor a } 3 \text { y APACHE } \\
\text { II mayor a } 8 \text { puntos }\end{array}$ \\
\hline
\end{tabular}

APACHE II: Acute Physiology and Chronic Health Evaluation II; ISSEMYM: Instituto de Seguridad Social del Estado de México y Municipios.

demostradas por aspiración de aguja fina o evidencia de gas en el contraste. La tomografía axial computarizada (TC) con medio de contraste puede confirmar las complicaciones intraabdominales, que incluyen necrosis infectada o colección infectada, infarto intestinal o perforación, hemorragia o síndrome compartimental abdominal. El papel de la cirugía en pacientes con necrosis estéril se discute de forma controvertida. Las terapias de intervención y la cirugía mínimamente invasiva han surgido en los últimos años, pero aún se limitan a los estudios clínicos en instituciones especializadas $^{7-10}$.

\section{Material y métodos}

Se realizó una revisión de los archivos clínicos e imagenológicos de pacientes con diagnóstico de PA grave (menor a seis semanas) que hayan sido sometidos algún tipo de tratamiento quirúrgico (abierto o laparoscópico) en el periodo comprendido entre el año 2013 y el 2019 en el Centro Médico ISSEMyM (Instituto de Seguridad Social del Estado de México y Municipios) en la población de Toluca, México.

Los criterios de inclusión y exclusión se muestran en la tabla 1.

Se estudiaron las variables cuantitativas discretas y nominales: edad, tasa de complicaciones y tasa de mortalidad. 


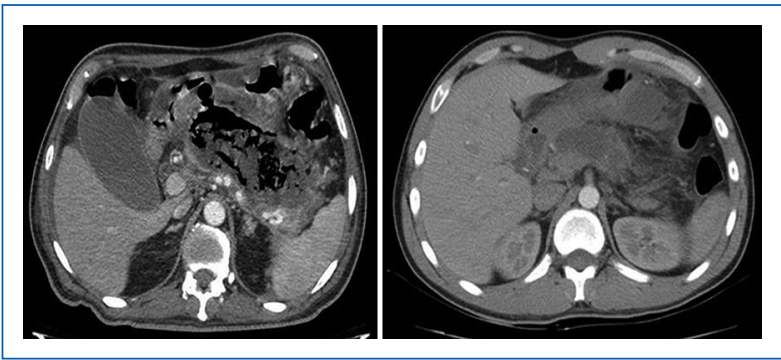

Figura 1. Tomografía axial computarizada simple (derecha) y contrastada izquierda. En corte axial donde se muestra (izquierda) abundante gas peripancreático y necrosis (flecha blanca). Derecha colección peripancreática (flecha amarilla).

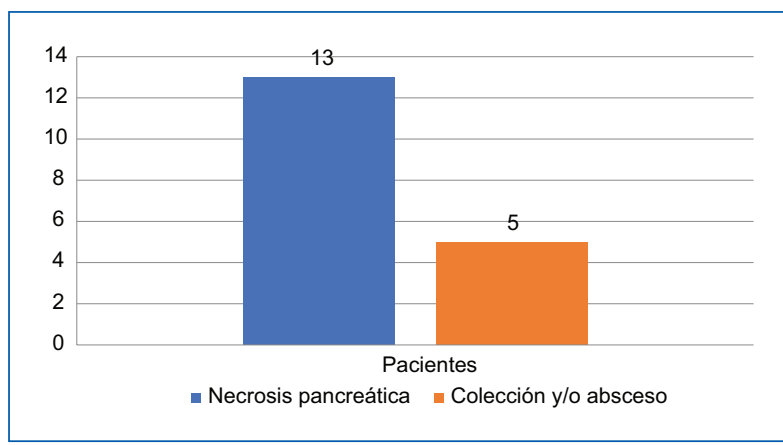

Figura 2. Complicaciones agudas más frecuentes.

Se estudiaron variables cualitativas nominales: sexo, etiología presuntiva, diagnóstico tomográfico con escala de Balthazar, tipo de abordaje (abierto o laparoscópico) y tipo de manejo transoperatorio (drenaje percutáneo, necrosectomía, drenaje y lavado quirúrgico, derivación y manejo de abdomen abierto con irrigación succión).

Estadísticamente se analizaron las variables preoperatorias: edad, comorbilidades, causa etiológica sugestiva de la pancreatitis y hallazgo tomográfico preoperatorio. También las variables transoperatorias y postoperatorias: abordaje abierto o laparoscópico, manejo con necrosectomía, drenaje, derivación o manejo de abdomen abierto. Así como las complicaciones más frecuentes y la tasa de mortalidad.

Los resultados se analizaron mediante estadística descriptiva no inferencial para el reporte de los resultados.

\section{Resultados}

Se identificaron un total de 59 pacientes con diagnóstico CIE-10 K85 de PA severa, de los cuales 30 pacientes fueron excluidos por no contar con los

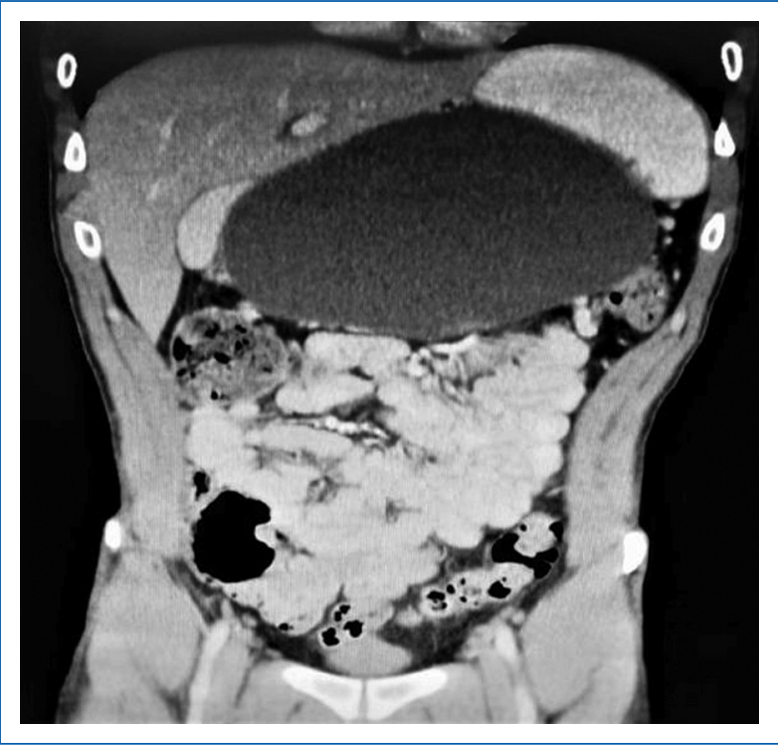

Figura 3. Tomografía axial computarizada en corte coronal donde se muestra un pseudoquiste pancreático de gran tamaño (flecha blanca).

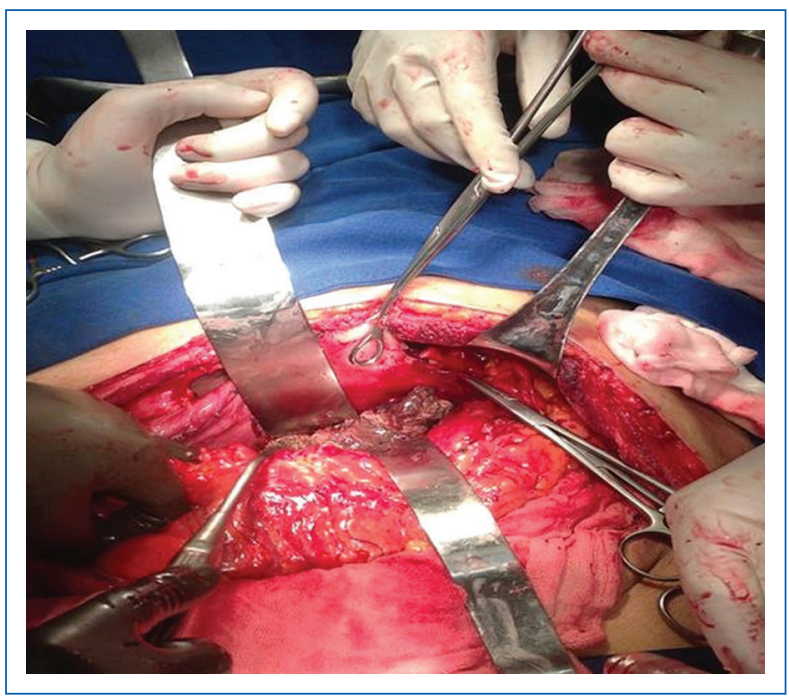

Figura 4. Laparotomía exploradora con abordaje tipo Chevron, donde se muestra la presencia de necrosis pancreática (flecha blanca).

requisitos establecido en la NOM-004-SSA1-2012 del expediente clínico.

Del total de 29 pacientes que se incluyeron en el estudio, 16 fueron hombres (56\%) y 13 mujeres (44\%). El rango de edad media se calculó en 45.5 años, con un rango de 24 a 91 años.

Del total de pacientes, 27 contaban con reporte de litiasis vesicular, estableciendo un $92.5 \%$ como etiología 


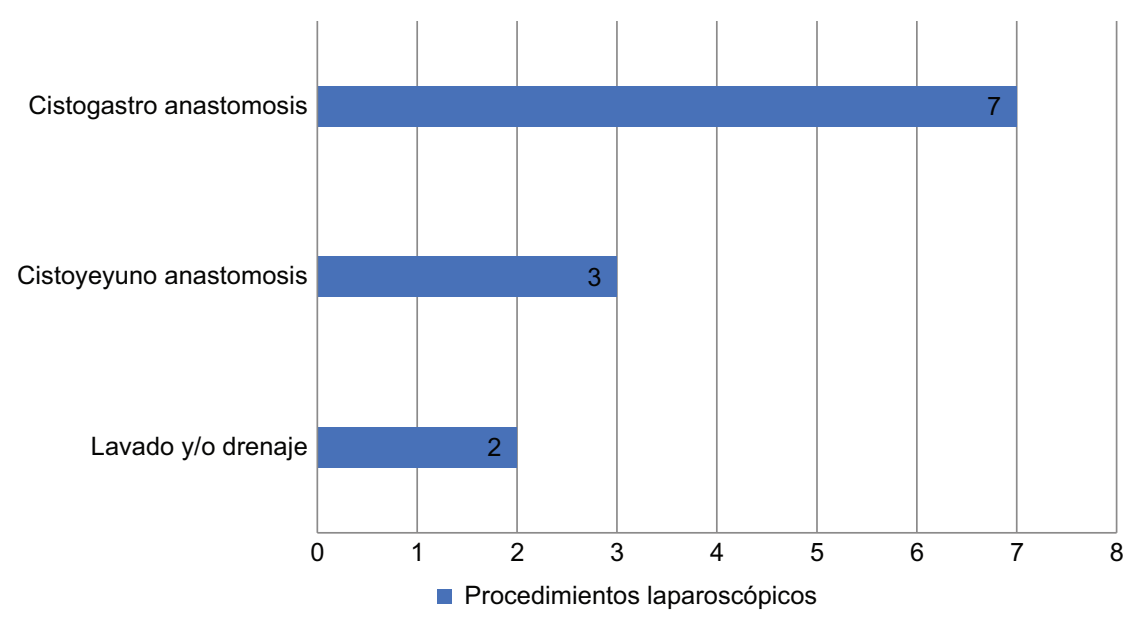

Figura 5. Procedimientos laparoscópicos realizados.

probable y 2 pacientes contaban con diagnóstico de hipertrigliceridemia (mayor a 1,000 g/dl), estableciendo solo el $7.4 \%$ para otras causas de pancreatitis.

A todos los pacientes (100\%) se les realizó TC toracoabdominopélvica con medio de contraste intravenoso de tres fases y oral (Fig. 1), cuyos diagnósticos fueron: 13 pacientes (44.44\%) con diagnóstico radiológico de necrosis pancreática con datos sugestivos de infección, 5 pacientes (18.5\%) con imagen sugestiva de colección y/o absceso peripancreático (Fig. 2); con escala de Balthazar grado E 13 pacientes y grado D, 5 pacientes.

Del total de pacientes con diagnóstico de necrosis pancreática y colección o absceso (18 pacientes en total), el $100 \%$ presentaron procalcitonina (PCT) con rango mayor a $2 \mathrm{ng} / \mathrm{dl}$, leucocitosis mayor a 12,000, fiebre y taquicardia como datos de respuesta inflamatoria sistémica.

Por otra parte, en 10 pacientes con diagnóstico inicial de PA severa posteriormente se estableció el diagnóstico radiológico de pseudoquiste pancreático (Fig. 3).

Del total de pacientes diagnosticados posteriormente con pseudoquiste pancreático, el $100 \%$ presentaba plenitud temprana, perdida ponderal y dolor abdominal con escala analógica del dolor 7 de 10.

Del total de pacientes diagnosticados se realizaron los siguientes procedimientos: el 55\% (14) fueron sometidos a laparotomía exploradora, el $44.3 \%$ (12) a laparoscopia exploradora y el $0.7 \%$ (2) a drenaje.

Del total de abordajes por laparotomía e intervencionismo, se realizaron: dos drenajes percutáneos, cuatro laparotomías con drenaje, lavado y manejo de abdomen abierto con sistema de presión negativa y 10 necrosectomías (Fig. 4), drenaje, lavado y cierre.

Del total de abordajes laparoscópicos, se realizaron: dos drenaje y lavado de cavidad, tres cistoyeyuno anastomosis y siete cistogastroyeyuno anastomosis (Fig. 5).

Como complicaciones se presentaron: un caso de síndrome compartimental que ameritó reintervención y manejo de abdomen abierto, una perforación intestinal que requirió la realización de colostomía y un paciente con fistula pancreática manejado con análogo de la octreótida con éxito, estableciendo una tasa de complicaciones del $11.1 \%$. Se reportaron dos defunciones por fracaso multiorgánico solamente durante los últimos seis años, como resultado, la tasa de mortalidad fue del $7.7 \%$

\section{Discusión}

La pancreatitis como patología frecuente es una de las causas más elevadas de morbimortalidad en los centros hospitalarios. Su diagnóstico oportuno, así como el tratamiento eficaz basado en las guías actuales sobre su abordaje son la clave principal en la evolución del paciente. Con base en las complicaciones inmediatas del cuadro, las patologías agregadas de índole infecciosa, como la necrosis o colecciones infectadas, obligan al especialista quirúrgico a la toma de decisiones rápidas y eficaces en el tratamiento; así mismo, el especialista debe de ser capaz de contar con los recursos profesionales y materiales para el tratamiento de las complicaciones. El tratamiento debe basarse siempre en la estadística actual, así como en 
la habilidad del cirujano, el cual dependerá de sus facultades al elegir un método abierto o laparoscópico para su resolución. En la actualidad la selección de pacientes con patologías pancreáticas agudas es de suma importancia para la elección del tipo de tratamiento. Si bien estudios comparativos inician en la actualidad para discernir sobre un abordaje o tratamiento quirúrgico, la mínima invasión (laparoscópica o por radiología intervencionista) nos otorga un panorama más amplio para el tratamiento de este tipo de pacientes.

\section{Conclusiones}

La PA y su evolución clínica se puede manifestar como: a) colección aguda y b) necrosis infectada y posteriormente como pseudoquiste pancreático.

En sus fases iniciales la presencia de respuesta inflamatoria y datos bioquímicos de infección como la PCT deben hacer plantear al cirujano la opción del tratamiento quirúrgico.

El manejo multidisciplinario coordinado y la temporización del tratamiento quirúrgico sugieren ser dos factores que cambian la evolución hacia favorable de estos casos.

Se prefiere el abdomen abierto para la fase aguda y la derivación laparoscópica para la fase crónica de las complicaciones, ya que han demostrado mejor control del daño y recuperación más rápida.

El manejo quirúrgico en pacientes con PA sigue siendo controvertido, por el riesgo de empeoramiento en el estado del paciente, pero debe ser un recurso disponible.

\section{Financiamiento}

Los autores declaran no haber recibido apoyo financiero o patrocinio de ninguna institución pública o privada.

\section{Conflicto de intereses}

Todos los autores declaran no tener conflicto de interés alguno.

\section{Responsabilidades éticas}

Protección de personas y animales. Los autores declaran que para esta investigación no se han realizado experimentos en seres humanos ni en animales.

Confidencialidad de los datos. Los autores declaran que han seguido los protocolos de su centro de trabajo sobre la publicación de datos de pacientes.

Derecho a la privacidad y consentimiento informado. Los autores han obtenido el consentimiento informado de los pacientes y/o sujetos referidos en el artículo. Este documento obra en poder del autor de correspondencia.

\section{Bibliografía}

1. Munigala S, Subramaniam D, Subramaniam DP, Buchanan $P$, Xian $H$, Burroughs $T$, et al. Predictors for early readmission in acute pancreatitis (AP) in the United States (US) - A nationwide population based study. Pancreatology. 2017;17(4):534-42.

2. Uc A, Fishman DS. Pancreatic disorders. Pediatr Clin North Am. 2017;64(3):685-706.

3. Bollen TL, van Santvoort HC, Besselink MG, van Leeuwen MS Horvath KD, Freeny PC, et al. The Atlanta Classification of acute pancreatitis revisited. Br J Sur. 2008;95:6-216.

4. Mofidi R, Patil PV, Suttie SA, Parks RW. Risk assessment in acute pancreatitis. Br J Surg. 2009:96:137-50.

5. Chen H, Li F, Sun JB, Jia JG. Abdominal compartment síndrome in patients with severe acute pancreatitis in early stage. World J Gastroenterol. 2008;14(22):3541-8.

6. Petrov MS, Shanbhag S, Chakraborty M, Phillips AR, Windsor JA. Organ failure and infection of pancreatic necrosis as determinants of mortality in patients with acute pancreatitis. Gastroenterology. 2010;139(3):813-20.

7. Buchler MW, Gloor B, Müller CA, Friess H, Seiler CA, Uhl W. Acute necrotizing pancreatitis: treatment strategy according to the status of infection. Ann Surg. 2000;232(5):619-26.

8. Hartwig W, Werner J, Müller CA, Uhl W, Büchler MW. Surgical management of severe pancreatitis including sterile necrosis. J Hepatobiliary Pancreat Surg. 2002;9(4):429-35.

9. van Sonnenberg E, Wittich GR, Chon KS, D'Agostino HB, Casola G, Easter D, et al. Percutaneous radiologic drainage of pancreatic abscesses. Am J Roentgenol. 1997;168(4):979-84.

10. Freeny PC, Hauptmann E, Althaus SJ, Traverso LW, Sinanan M. Percutaneous CT-guided catheter drainage of infected acute necrotizing pancreatitis: techniques and results. Am J Roentgenol. 1998;170(4):969-75. 